\title{
Detection Error Aware Spectrum Handoff Mechanism for Cognitive Radios
}

\author{
Pedro Smith Coutinho, Marcel William Rocha da Silva and José Ferreira de Rezende \\ Universidade Federal do Rio de Janeiro \\ E-mails: \{coutinho, marcel, rezende\}@land.ufrj.br
}

\begin{abstract}
Spectrum handoff is a key mechanism for proper and efficient operation of cognitive radios. A handoff occurs when the current operating channel must be freed by secondary users due to the arrival of a primary user. This mechanism is responsible for searching for a new idle licensed channel for secondary use, a task known as channel selection. The order in which the channels are sensed during the handoff has a great impact on performance. This paper proposes a new spectrum handoff scheme that considers the existence of errors in primary user detection to achieve a better channel ordering in terms of spectrum utilization efficiency and primary user interference. Simulation results show that the proposed mechanism outperforms other mechanisms from the literature.

Index Terms-Cognitive radio, spectrum handoff, channel selection, spectrum sensing.
\end{abstract}

\section{INTRODUCTION}

Opportunistic spectrum usage by cognitive radios (CRs) [1], [2], [3] requires that these devices continuously select the most appropriate spectrum band to be used. Generally, the channel selection process, also called spectrum decision [3], improves as more spectrum opportunities are detected as accurately as possible. However, in CRs equipped with only one radio interface, the detection of opportunities through sensing incurs in a waste in the use of the discovered opportunities. Therefore, there is a strong coupling between spectrum sensing and spectrum decision schemes. To this end, several studies propose decision processes associated with sensing schemes that jointly seek to optimize the use of opportunities [4], [5], [6], [7], [8], [9].

The spectrum decision process is called handoff when the CR decides to switch the operating spectrum band. Spectrum handoff mechanisms are categorized as reactive or proactive. In reactive handoff, a secondary user (SU) searches for another channel only when primary user (PU) activity is detected in its current operating channel. In this case, the SU senses other channels in order to change to a new primary idle channel. Thus, the SU must periodically interrupt its transmission to sense the current operating channel to verify whether a PU has arrived and the channel must be freed or the secondary communication may continue on this same channel.

On the other hand, proactive mechanisms may be used to achieve different objectives. In the case where SUs need as much bandwidth as possible, periodic sensing of the other channels enables CRs to continuously discover new opportunities and thus obtain higher transmission rates by aggregating multiple idle channels [4]. The periodic sensing of the remaining spectrum bands may also allow the SUs to switch channels before the arrival of a PU. This early handoff may be related to multiple factors, such as the discovery of a channel with better conditions or with a higher probability of being idle for a longer timespan. Periodically sensing the remaining channels also enables SUs to order channels according to some criteria in preparation for a future handoff operation. This would allow SUs to optimize their performance in terms of spectrum utilization depending on the criterion used in the channel ordering.

The work in [4] proposes as ordering criterion the idle probability of each channel. In [8], the authors extend this proposal by taking into account the expected transmission time, proposed in [5], and the average idle durations of each channel. However, these works do not consider the probability of misdetection of the PU presence. In this paper, we propose a new channel ordering that takes this probability into account. Then, we have developed a simulation framework in order to compare all these proposals under the same conditions. Results show that the proposed criterion achieves a similar performance in terms of opportunity usage efficiency, while causing less interference to PUs.

This paper is organized as follows. Section II discusses previous work related to spectrum handoff. In Section III, we describe the SU behavior and licensed channel activity models. Section IV presents the spectrum handoff mechanism proposed in this paper. In Section V, we present the simulation environment used in the performance evaluation of the mechanisms, testing scenarios and the simulation results. Finally, we present conclusions and describe future work in Section VI.

\section{RELATED WORK}

The work in [4] assumes SUs are equipped with only one radio interface and perform periodic proactive sensing. It proposes the optimization of the sensing period in each channel in order to maximize the discovery of spectrum opportunities and to mitigate the overhead caused by proactive sensing. Moreover, the authors define a channel ordering algorithm that reduces the search latency of a new channel when a handoff occurs. In this algorithm, when a PU is detected, the channel with higher probability of being idle is chosen as the first candidate to be sensed. The work in [8] extends this algorithm combining this idle probability with the average idle time duration and expected transmission time. 
In [7], the authors propose two spectrum decision schemes. The first one selects spectrum bands that offer minimum variation in capacity for use in real-time applications. The second one seeks to maximize the network total capacity for best-effort applications. In addition to the spectrum decision schemes, the paper proposes dynamic resource management in order to coordinate decisions according to the bandwidth fluctuations caused by the arrival of PUs and/or SUs.

The work in [6] compares the performance of three spectrum handoff schemes through an analytical model. In the first scheme, SUs do not initiate handoff when a PU arrives, but only pause their transmission until the PU leaves the channel. The other two schemes are, respectively, proactive and reactive handoff methods. In the proactive scheme, an SU sorts the candidate channels to form an ordered list prior to the arrival of a PU. When a PU arrives, the SU uses the first channel in the list for the handoff. The channel list formation is based exclusively in channel state prediction models, which does not take sensing time into account and is prone to prediction errors. In the reactive scheme, the SU searches for an idle channel only after the arrival of a PU.

In [10], spectrum handoff is discussed in the scope of simultaneous multiple channel cognitive radio networks and delay sensitive communications, as for example VoIP. The authors propose PU behavior prediction and the use of backup channels in order to minimize handoff latency and mitigate the problems caused by prediction errors, which are unavoidable.

In [11], proactive and reactive sensing techniques for spectrum handoff are discussed. This work uses Queueing Theory to provide a Preemptive Resume Priority M/G/1 queueing network model of the primary and secondary networks and compare both techniques. The objective is to find out under which conditions each scheme is more appropriate. However, this work does not consider that the parameters of primary user activity must be estimated and that this generally implies in periodic sensing, which has a non-negligible cost. In [12], the same authors present a new proactive sensing channel selection mechanism with the objective of reducing the total service time of the network. The presented results consist of the comparison of the proposed mechanism with random channel selection.

The work in [13] analyses spectrum handoff in two scenarios. The first one is called opportunistic, in which there is no central entity that coordinates the SUs. The second one is called negotiated, in which there is a central server that manages the spectrum and coordinates SUs. Using Queueing Theory, both mechanisms are compared and the authors conclude that opportunistic handoff is superior in terms of success probability, defined as the probability of finding an idle channel, although it implies in a greater number of handoff operations than in the negotiated mechanism.

\section{SySTEM MODEL}

The system model adopted in this paper assumes a licensed band consisting of $N$ channels with bandwidth $W$. Due to PU activity, each licensed channel has an utilization pattern that can be represented by a continuous time two-state Markov chain. In this model, the $O N$ and $O F F$ states represent, respectively, the periods of activity (busy channel) and inactivity (idle channel) of the PU. The durations of the ON and OFF states, $t_{O N}$ and $t_{O F F}$, are given by exponentially distributed random variables with rates $\alpha$ and $\beta$, respectively.

This $\mathrm{ON}-\mathrm{OFF}$ licensed channel utilization model is adopted in several other works in the literature [4], [5], [14]. Moreover, according to the experimental results presented in [15], this model is valid in several different scenarios.

The secondary network considered in the model is infrastructured and may be thought of as a "cell", in which a base station (BS) coordinates access of the clients to licensed spectrum opportunities. In this scenario, client devices possess only minimal communication functionality due to hardware restrictions, such as low processing power and limited energy consumption. Therefore, all cognitive functionality is centralized and implemented by the BS, from PU detection to licensed band access opportunities discovery. Thus, the concepts of SU and secondary network are used as synonyms.

SU operation over time is shown in Fig 1. This model assumes that the secondary network makes use of only one channel at a time for its communication. To avoid causing interference to the PUs, the SU must periodically sense its current operating channel, represented by the short gray regions in the figure. During these periods, the BS makes sure all clients remain silent to avoid the influence of SU transmissions on the sensing. Hence, careful choice of sensing intervals in the operating channel plays an important role in the operation of the secondary network, as it represents a compromise between channel utilization efficiency and quick PU detection.

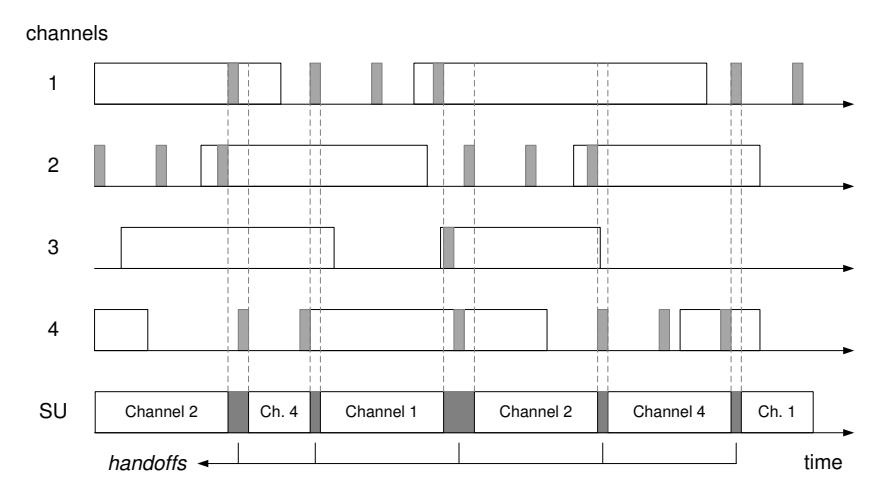

Fig. 1. Operation of SU over time.

Whenever the SU detects the arrival of a PU through sensing, the SU must start a spectrum handoff operation (dark gray regions in the SU timeline in Fig 1). In this phase, the SU sequentially senses the remaining channels of the licensed spectrum in order to find a new idle channel. The time spent to discover an idle channel is related to the ordering used to sequentially sense these spectrum bands. A good sensing order enables a quick discovery of an idle channel and/or guarantees that this channel will remain idle for an extended period of time. Thus, the overhead caused by the latency and number of handoff operations may be reduced. 
In addition to periodic sensing of the current operating channel, our system model assumes that the SU also periodically and proactively senses the remaining channels of the licensed spectrum. The objective of this sensing is to collect information that allows the SU to better determine the channel sensing order it will use during handoff operations.

\section{Proposed Mechanism}

The proposed spectrum handoff mechanism is divided in three parts: A) optimal sensing cycle calculation; B) proactive spectrum sensing; and C) sensing sequence determination. Each one of these parts is detailed in the following subsections.

\section{A. Optimal Sensing Cycle Calculation}

The sensing cycle determines the interval used by an SU between spectrum sensing operations in order to detect a possible PU arrival. According to the work in [5], there is an optimal sensing cycle for which maximum transmission time is achieved with limited interference to the PUs. The sensing cycle is given by equation 1 . It consists of the sum of sensing time $\left(t_{s}\right)$ and transmission time $\left(t_{t}\right)$. The optimal cycle value depends on factors such as the licensed channel activity model, the probability of false alarm in PU detection, among others.

$$
t_{c, i}=t_{s, i}+t_{t, i}
$$

In the proposed mechanism, the optimal sensing cycle of channel $i$ is calculated according to equations 1 to 3 , as in [5]. Equations 2 and 3 provide the sensing and transmission times of channel $i$, respectively.

$$
\begin{aligned}
& t_{s, i}=\frac{1}{W \cdot \gamma^{2}}\left[Q^{-1}\left(P_{F A}\right)+\right. \\
&\left.(1+\gamma) Q^{-1}\left(\frac{P_{O F F} P_{F A}}{P_{O N}}\right)\right]^{2}
\end{aligned}
$$

where $W$ is the channel bandwidth, $\gamma=\sigma_{x}^{2} / \sigma_{w}^{2}$ is the signalto-noise ratio (SNR), $Q^{-1}$ is the inverse gaussian $Q$ function, $P_{F A}$ is the false alarm probability and $P_{O F F}$ and $P_{O N}$ are the probabilities that channel $i$ is idle and busy, respectively.

$$
t_{t, i}=-\frac{1}{\mu} \cdot \log \left(1-\frac{P_{I}}{P_{O F F}}\right)
$$

where $\mu=\max (\alpha, \beta), \alpha=\frac{1}{t_{O N}}, \beta=\frac{1}{t_{O F F}}$ and $P_{I}$ is the maximum interference rate to be caused to PUs.

In order to calculate these values, a channel activity estimator was needed, and it was implemented as in [4]. The estimator assumes that all channels follow an $\mathrm{ON}-\mathrm{OFF}$ activity pattern with exponentially distributed state durations.

\section{B. Proactive Spectrum Sensing}

In addition to periodically sensing the current operating channel, the proposed mechanism relies on the sensing of the $N-1$ remaining channels. This enables the SU to estimate each channel's activity model, through which it will be possible to calculate the optimal sensing cycle. Furthermore, it also enables the SU to sample the channels' states to aid in the determination of the handoff sensing sequence, described in the next subsection. Proactive sensing forces the SU to interrupt its transmission in order to perform this task, since it is equipped with only one radio interface. Therefore, proactive sensing incurs in a waste in the use of spectrum opportunities, but it may bring benefits when handoffs need to be performed.

A more frequent proactive spectrum sensing will result in a greater overhead, but also in more accurate information. When the proactive sensing interval is too large, the channel activity parameter estimates become inaccurate, negatively impacting the performance of the handoff mechanism.

\section{Handoff Sensing Sequence}

The handoff sensing sequence is the order in which the remaining channels will be sensed by the SU when searching for a new idle channel. In order to obtain this sequence, the SU needs to know each channel's activity model and the state in which it was when it was last sensed. All originality of the proposed mechanism lies in this part of the task.

The sensing sequence determined by the proposed mechanism takes into account the channel's probability of being idle $\left(P_{I D L E}\right)$ and the expected transmission and interference times of such channel. The probability $P_{I D L E}$, computed by equation 6 , uses the state transition probabilities $P_{00}(\Delta t)$ and $P_{10}(\Delta t)$ to establish a relation between channel $i$ 's state sampled at a given point in time with its probability of being idle at $\Delta t$ after the sample has been collected [4]. Equations 4 and 5 show the probability that channel $i$ is idle $\Delta t$ after being sampled as idle and busy, respectively.

$$
\begin{aligned}
& P_{00, i}(\Delta t)=\left(1-P_{O N, i}\right)+P_{O N, i} \cdot e^{-\left(\alpha_{i}+\beta_{i}\right) \Delta t} \\
& P_{10, i}(\Delta t)=\left(1-P_{O N, i}\right)+\left(1-P_{O N, i}\right) \cdot e^{-\left(\alpha_{i}+\beta_{i}\right) \Delta t}
\end{aligned}
$$

$$
P_{I D L E, i}(\Delta t)=\left\{\begin{array}{l}
P_{00, i}(\Delta t), \text { if } d_{i}=0 \\
P_{10, i}(\Delta t), \text { if } d_{i}=1
\end{array}\right.
$$

where $d_{i}$ is the state of channel $i$ in the last collected sample and $\Delta t$ is the time elapsed between the collection of this sample and the handoff operation.

The expected transmission time $\left(t_{E}\right)$, as defined in [7] and also used in [8], is the timespan, during an idle period of channel $i$, from the start of the SU transmission to the need of a handoff operation. This handoff may occur due to either the actual arrival of a PU or a false alarm in its detection. The expected transmission time $t_{E}$ is given by equation 7 .

$$
\begin{aligned}
t_{E, i}=t_{c, i} & \cdot \sum_{k=1}^{n-1} k \cdot\left(1-P_{F A}\right)^{k} \cdot P_{F A}+\frac{1}{\beta_{i}}\left(1-P_{F A}\right)^{n} \\
=t_{c, i} & \cdot\left[\frac{\left(1-P_{F A}\right)\left(1-\left(1-P_{F A}\right)^{n-1}\right)}{P_{F A}}\right. \\
& \left.-(n-1) \cdot\left(1-P_{F A}\right)^{n}\right]+\frac{1}{\beta_{i}}\left(1-P_{F A}\right)^{n}
\end{aligned}
$$


where $n=\left\lceil 1 / \beta_{i} / t_{c, i}\right\rceil$ is the average number of sensing cycles in channel $i$ 's idle period and $\frac{1}{\beta_{i}}=t_{O F F, i}$ is the duration of channel $i$ 's idle period.

The expected interference time $\left(t_{I}\right)$, defined in this paper, considers the detection error probability in the arrival of a PU. It corresponds to the timespan, during a busy period of channel $i$, between the arrival of a PU and its correct detection, as shown in equation 8 .

$$
\begin{aligned}
t_{I, i}=t_{c, i} & \cdot \sum_{k=1}^{m-1} k \cdot P_{M D}{ }^{k} \cdot\left(1-P_{M D}\right)+\frac{1}{\alpha_{i}} P_{M D}{ }^{m} \\
=t_{c, i} & \cdot\left[\frac{P_{M D}\left(1-P_{M D}{ }^{m-1}\right)}{\left(1-P_{M D}\right)}\right. \\
& \left.-(m-1) \cdot P_{M D}^{m}\right]+\frac{1}{\alpha_{i}} P_{M D}^{m}
\end{aligned}
$$

where $m=\left\lceil 1 / \alpha_{i} / t_{c, i}\right\rceil$ is the average number of sensing cycles in channel $i$ 's busy period and $\frac{1}{\alpha_{i}}=t_{O N, i}$ is the duration of channel $i$ 's busy period.

The channel ordering criterion proposed in this paper combines the channel's probability of being idle $\left(P_{I D L E}\right)$ and expected transmission time $\left(t_{E}\right)$ with the expected interference time $\left(t_{I}\right)$, as shown in equation 9 . The objective is to sort channels by greater $P_{I D L E}$ and $t_{E}$, and lower $t_{I}$, simultaneously.

$$
C=\frac{P_{I D L E} \cdot t_{E}}{t_{I}}
$$

\section{Simulation and Numeric Results}

For the performance evaluation presented in this paper, the proposed mechanism, among with three other existing handoff schemes from the literature, were implemented in a discrete event simulator. The common part of the simulator implements the channel activity models, optimal sensing cycle calculation and periodic proactive sensing of channels. The specific part to each mechanism involves the spectrum sensing sequence ordering which is used by the SU when a handoff occurs.

TABLE I

IMPLEMENTED HANDOFF SCHEMES

\begin{tabular}{|l|c|c|}
\hline Scheme & Criterion & Citation \\
\hline \hline kim & $P_{I D L E}$ & {$[4]$} \\
\hline duan & $P_{I D L E} \cdot t_{E} \cdot T_{d}\left(t_{E}\right)$ & {$[8]$} \\
\hline lee & $t_{E}$ & {$[7]$} \\
\hline proposed & $\frac{P_{I D L E} \cdot t_{E}}{t_{I}}$ & - \\
\hline
\end{tabular}

The difference between the evaluated mechanisms is the criteria used for the channel ordering. Table I shows all of the implemented handoff mechanisms with their respective ordering criteria and citation.

For the scheme named duan, the authors derive the $T_{d}(t, i)$ function which provides the expected value of the amount of time $t$ that channel $i$ will remain idle [8]. This function is used as one of the terms in the channel ordering criterion with the expected transmission time $t_{E}$ as its input parameter.

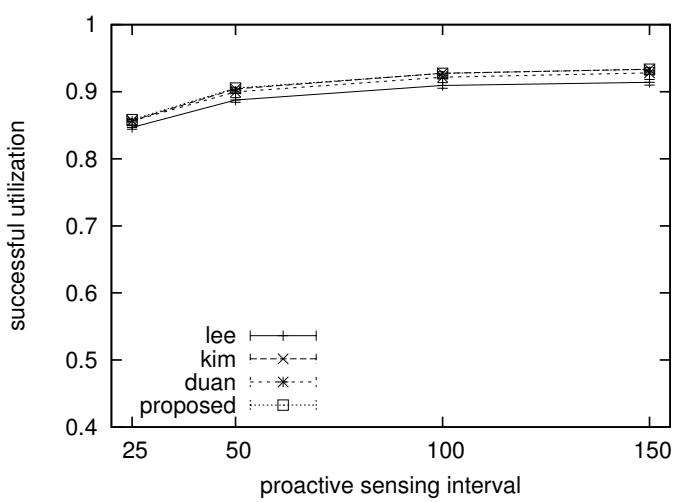

(a) successful utilization

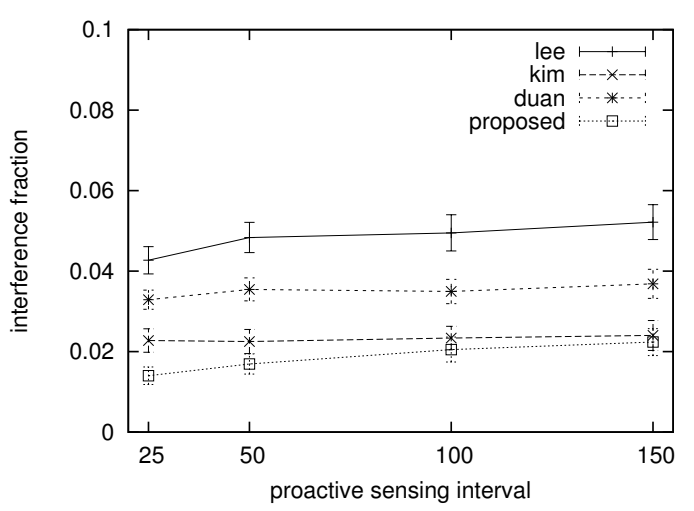

(b) interference fraction

Fig. 2. $t_{\text {pscan }}$ variation for $P_{M D}=0.1$ and $t_{s w}=0.15 \mathrm{~s}$.

Each simulation round lasted for 100000s and 50 rounds were run for each parameter set. There were 15 licensed channels and only one SU (or secondary network). The simulation parameters are described in Table II.

TABLE II

Simulation PARAMETERS

\begin{tabular}{|l|c|c|}
\hline Param. & Description & Value \\
\hline \hline$W$ & channel bandwidth & $10 \mathrm{KHz}$ \\
\hline$\gamma$ & primary signal SNR & $-5 \mathrm{~dB}$ \\
\hline$t_{\text {pscan }}$ & proactive sensing interval & $25,50,100,150 \mathrm{~s}$ \\
\hline$P_{I}$ & max. primary interference rate & 0.05 \\
\hline$P_{F A}$ & false alarm probability & 0.01 \\
\hline$P_{M D}$ & PU misdetection probability & $0.01,0.1,0.2$ \\
\hline$t_{\text {sw }}$ & channel switching delay & $0.015,0.15,0.5,1.0,1.5 \mathrm{~s}$ \\
\hline$t_{O F F}$ & average channel busy time & uniformly distr. [5-100] $\mathrm{s}$ \\
\hline$t_{O N}$ & average channel idle time & uniformly distr. [5-100] $\mathrm{s}$ \\
\hline
\end{tabular}

The performance metrics used in the evaluation are successful spectrum utilization and PU interference fraction. These metrics correspond to the fractions of time in which the SU uses the licensed spectrum with and without causing interference to the PUs, respectively. All presented results correspond to the average of the 50 simulation rounds and the error bars correspond to $95 \%$ confidence intervals.

Figs 2 and 3 present the results of successful spectrum utilization and PU interference fraction over the variation of 


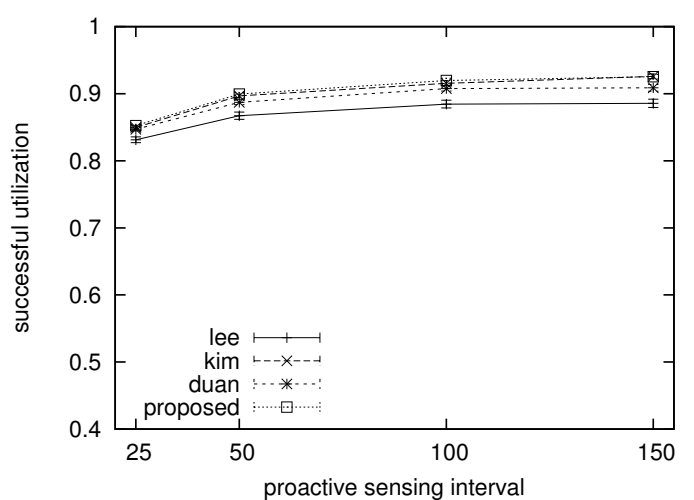

(a) successful utilization

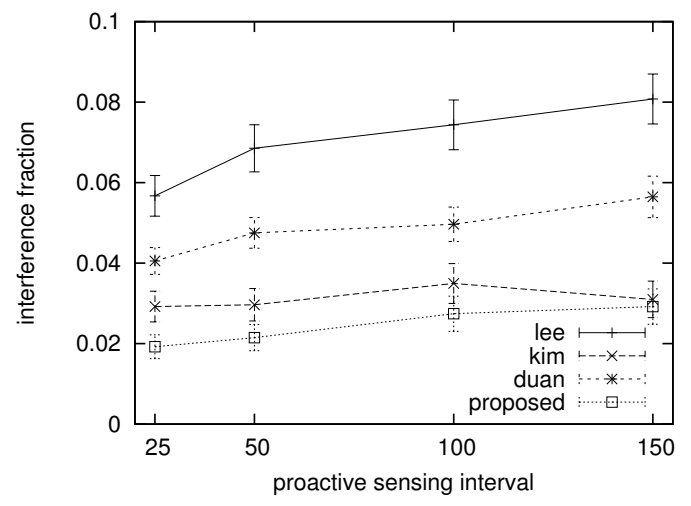

(b) interference fraction

Fig. 3. $t_{\text {pscan }}$ variation for $P_{M D}=0.2$ and $t_{s w}=0.15 \mathrm{~s}$.

the proactive scanning intervals $\left(t_{\text {pscan }}\right)$. The probability of misdetection $P_{M D}$ was set to 0.1 in Fig 2 and 0.2 in Fig 3. In both scenarios, $t_{s w}$ was set to $0.15 \mathrm{~s}$.

The results show that in both scenarios and for all scanning intervals, the performance of the proposed mechanism is very close to all other mechanisms except for lee. This happens because all sensing intervals used have enabled these schemes, which are based on previous channel states, to gather enough updated information, having an advantage over it.

Still regarding these results, it is important to notice that the proposed handoff scheme performs considerably better in terms of interference fraction when compared to the remaining schemes. The better performance is explained by the fact that it is the only mechanism to consider the expected interference time $\left(t_{I}\right)$ in the handoff channel ordering. This demonstrates the importance of this parameter in scenarios where the probability of misdetection $\left(P_{M D}\right)$ is not negligible.

Figs 4 and 5 present the results of the evaluated schemes over the variation of the channel switching delay $\left(t_{s w}\right)$. It can be noticed that the proposed handoff scheme has the lowest interference fraction for all switching delay values. This occurs because it considers the expected interference time $\left(t_{I}\right)$ in the channel ordering. In addition, one can see that a greater $t_{s w}$ causes smaller successful utilization and interference fraction in all mechanisms. The main reason for this is the increase

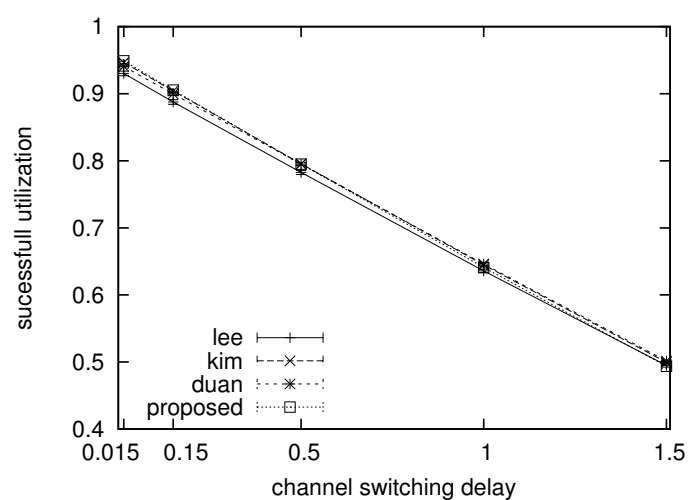

(a) successful utilization

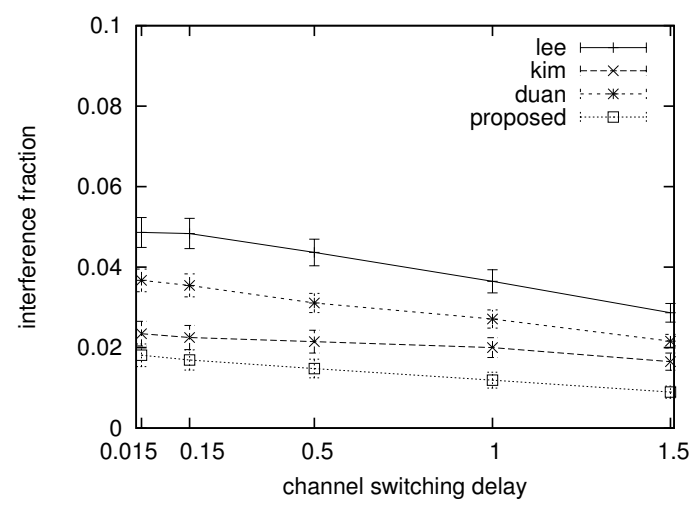

(b) interference fraction

Fig. 4. $t_{s w}$ variation for $P_{M D}=0.1$ and $t_{\text {pscan }}=50 \mathrm{~s}$.

of the channel switching overhead, which becomes highly significant in comparison to the remaining SU transmission time, either with success or causing interference. It is worth mentioning that, in addition to the channel switching performed during handoff, the periodic scanning of the $N-1$ remaining channels also incurs in channel switching, adding to the already greater overhead.

Fig 6 shows the influence of the probability of misdetection $\left(P_{M D}\right)$ on spectrum utilization and interference fraction. As expected, as this parameter increases, the interference caused on PUs is greater (Fig 6(b)). However, as the proposed mechanism takes this factor into account on its channel sensing ordering, it provides the lowest interference fraction when compared to the other mechanisms. Regarding successful spectrum utilization, an increase in $P_{M D}$ has a greater impact on the lee scheme since this mechanism favors channels with higher expected transmission times, which in consequence may suffer interference for longer durations.

\section{CONCLUSIONS AND FUTURE WORK}

Spectrum handoff is an important mechanism in the performance of cognitive radios. It not only determines when the SU should switch its operating channel, but towards which channel the switch should be made. Mechanisms that perform proactive spectrum sensing are able to maintain an ordered 


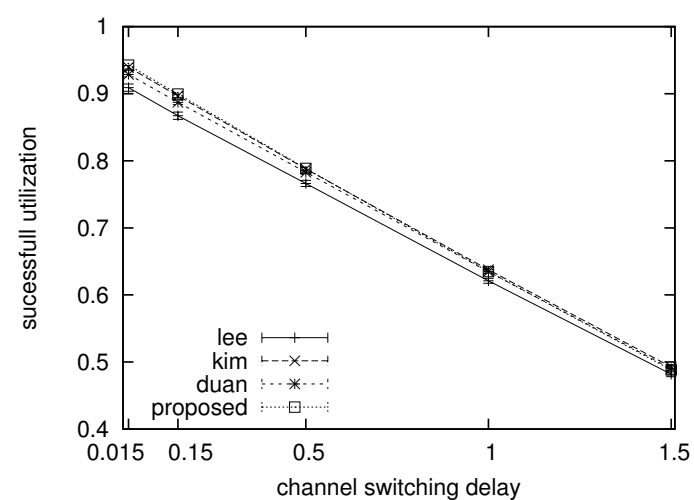

(a) successful utilization

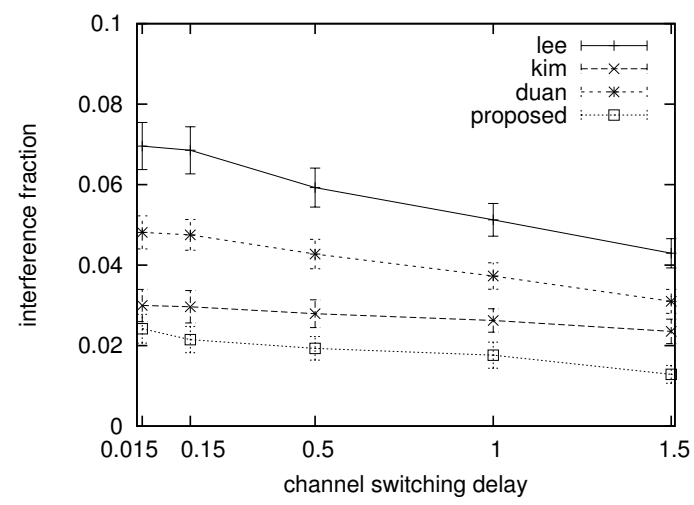

(b) interference fraction

Fig. 5. $t_{s w}$ variation for $P_{M D}=0.2$ and $t_{\text {pscan }}=50 \mathrm{~s}$.

list of channels according to the preference of handoff. In such a way, when a SU must free a licensed channel due to the arrival of a PU, this list may be used in order to more efficiently decide which channel to use from that point on.

This paper proposes a new criterion for channel ordering that takes the expected interference to be caused on primary users into account. This proposed mechanism was compared against other mechanisms found in the literature, and the results demonstrate its ability to ensure good spectrum utilization while keeping interference at a limited level, even in scenarios with high primary user misdetection probability $\left(P_{M D}\right)$.

As future work, new channel ordering criteria can be elaborated. Furthermore, channel activity prediction schemes could be used to assist handoff mechanisms.

\section{REFERENCES}

[1] J. Mitola III, "Cognitive radio: Model-based competence for software radios," Ph.D. dissertation, Dept. of Teleinformatics, KTH, 1999.

[2] S. Haykin, "Cognitive radio: brain-empowered wireless communications," IEEE Journal on Selected Areas in Communications, vol. 23, no. 2, pp. 201-220, Feb. 2005.

[3] I. F. Akyildiz, W.-Y. Lee, M. C. Vuran, and S. Mohanty, "NeXt generation/dynamic spectrum access/cognitive radio wireless networks: a survey," Computer Networks, vol. 50, pp. 2127-2159, Sep. 2006.

[4] H. Kim and K. G. Shin, "Efficient discovery of spectrum opportunities with MAC-layer sensing in cognitive radio networks," IEEE Transactions on Mobile Computing, vol. 7, no. 5, pp. 533-545, May 2008.

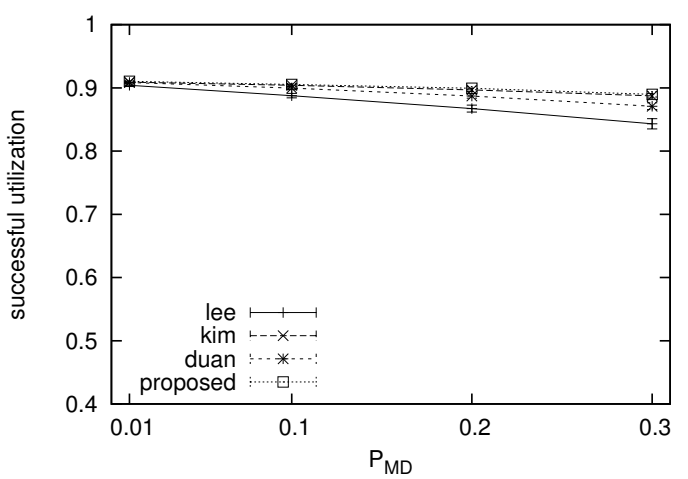

(a) successful utilization

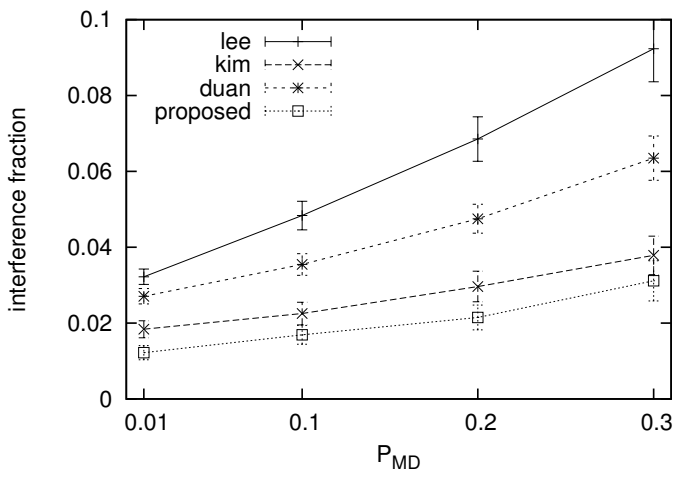

(b) interference fraction

Fig. 6. $P_{M D}$ variation for $t_{s w}=0.15 \mathrm{~s}$ and $t_{\text {pscan }}=50 \mathrm{~s}$.

[5] W.-Y. Lee and I. F. Akyildiz, "Optimal spectrum sensing framework for cognitive radio networks," IEEE Transactions on Wireless Communications, vol. 7, no. 10, pp. 3845-3857, Oct. 2008.

[6] L.-C. Wang and C. Anderson, "On the performance of spectrum handoff for link maintenance in cognitive radio," in Proceedings of ISWPC, May 2008, pp. 670-674.

[7] W.-Y. Lee and I. F. Akyildiz, "A spectrum decision framework for cognitive radio networks," IEEE Transactions on Mobile Computing, vol. 10, no. 2, pp. 161-174, Feb. 2011.

[8] J. Duan and Y. Li, "An optimal spectrum handoff scheme for cognitive radio mobile ad hoc networks," Advances in Electrical and Computer Engineering, vol. 11, no. 3, pp. 11-16, May 2011.

[9] M. Hoyhtya, S. Pollin, and A. Mammela, "Classification-based predictive channel selection for cognitive radios," in Proceedings of ICC, May 2010, pp. 1-6.

[10] A. Lertsinsrubtavee, N. Malouch, and S. Fdida, "Spectrum handoff strategies for multiple channels cognitive radio network," in Proceedings of the ACM CoNEXT Student Workshop 2010, Nov. 2010, pp. 20:1-20:2.

[11] L.-C. Wang and C.-W. Wang, "Spectrum handoff for cognitive radio networks: Reactive-sensing or proactive-sensing?" in Proceedings of IEEE IPCCC, Dec. 2008, pp. 343-348.

[12] C.-W. Wang and L.-C. Wang, "Modeling and analysis for proactivedecision spectrum handoff in cognitive radio networks," in Proceedings of IEEE ICC, Jun. 2009, pp. 1-6.

[13] Y. Zhang, "Spectrum handoff in cognitive radio networks: Opportunistic and negotiated situations," in Proceedings of IEEE ICC, Jun. 2009, pp. $1-6$.

[14] M. W. R. da Silva and J. F. de Rezende, "Routing in hybrid mesh networks of cognitive and IEEE 802.11 radios," in Proceedings of IEEE GLOBECOM, Dec. 2010, pp. 1-5.

[15] M. Wellens, J. Riihijarvi, and P. Mahonen, "Empirical time and frequency domain models of spectrum use," Physical Communication, vol. 2, no. 1-2, pp. 10-32, 2009. 